\title{
A Model for a Non-Minimally Coupled Scalar Field Interacting with Dark Matter
}

\author{
J. B. Binder and G. M. Kremer \\ Departamento de Física, Universidade Federal do Paraná \\ Caixa Postal 19044, 81531-990 Curitiba, Brazil
}

(Received on 14 October, 2005)

\begin{abstract}
In this work we investigate the evolution of a Universe consisted of a scalar field, a dark matter field and noninteracting baryonic matter and radiation. The scalar field, which plays the role of dark energy, is non-minimally coupled to space-time curvature, and drives the Universe to a present accelerated expansion. The non-relativistic dark matter field interacts directly with the dark energy and has a pressure which follows from a thermodynamic theory. We show that this model can reproduce the expected behavior of the density parameters, deceleration parameter and luminosity distance.
\end{abstract}

\section{INTRODUCTION}

Recently, well known astronomical observations with super-novae of type Ia suggested that our Universe is presently submitted to an accelerated expansion $[1,2]$ but the nature of the responsible entity, the so-called dark energy, still remains unknown. The simplest theoretical explanation for the acceleration consists in introducing a cosmological constant and investigating the so-called $\Lambda C D M$ model (see [3]), which fits the present data very well but has some important unsolved problems. Another possibility is to introduce a minimally coupled scalar field $\phi(t)$, which has been extensively studied by the scientific community and could be invalidated if future observations imply that the ratio between the pressure and the energy density of the scalar field is restricted to values $p_{\phi} / \rho_{\phi}<-1$ (see [4]).

In this work we consider a scalar field non-minimally coupled to space-time curvature, which was investigated in [5] and widely studied recently, among others, in the works [612]. Also, aside several models for the dark sector interaction [13-15], we consider here a direct coupling between dark matter and dark energy, following a model proposed in [16] and studied more recently in [17] and [18]. We also use a thermodynamic theory [19] in order to relate the effects of the non-minimally coupling to the dark matter pressure [12]. All components will be described by a set of field equations, and the resulting observables - namely, the density parameters, the decelerating parameter and the luminosity distance which are obtained as solutions of the field equations, will be compared to the available data set in order to draw the conclusions about the viability of this model. We show that physically acceptable solutions are obtained and that there exist some freedom parameters that will be important to fit the data from incoming experiments. Units have been chosen so that $8 \pi G=c=\hbar=k=1$, whereas the metric tensor has signature $(+,-,-,-)$.

\section{FIELD EQUATIONS}

We shall model the Universe as a mixture of a scalar field, a dark matter field and non-interacting baryons and radiation. Furthermore, we shall consider that the scalar field, which plays the role of dark energy, is non-minimally coupled to the scalar curvature [5] and that the dark matter interacts with the scalar field according to a model proposed by Wetterich [16, 17].

For an isotropic, homogeneous and spatially flat Universe described by the Robertson-Walker metric

$$
d s^{2}=d t^{2}-a(t)^{2}\left(d x^{2}+d y^{2}+d z^{2}\right),
$$

with $a(t)$ denoting the cosmic scale factor, the Friedmann and acceleration equations read

$$
H^{2}=\frac{\rho}{3\left(1-\xi \phi^{2}\right)}, \quad \frac{\ddot{a}}{a}=-\frac{\rho+3 p}{6\left(1-\xi \phi^{2}\right)},
$$

respectively. The quantities $\rho=\rho_{b}+\rho_{r}+\rho_{d m}+\rho_{\phi}$ and $p=p_{b}+p_{r}+p_{d m}+p_{\phi}$ denote the energy density and the pressure of the sources of the gravitational field, respectively, and they are given in terms of the respective quantities for its constituents. Moreover, the dot denotes the differentiation with respect to time, $H=\dot{a} / a$ is the Hubble parameter, and $\xi$ the coupling constant between the scalar field $\phi$ and the curvature scalar.

The modified Klein-Gordon equation for the scalar field, which takes into account the non-minimal coupling and the interaction with the dark matter, reads

$$
\ddot{\phi}+3 H \dot{\phi}+\frac{d V}{d \phi}+\frac{\xi \phi}{1-\xi \phi^{2}}(\rho-3 p)-\beta \rho_{d m}=0,
$$

where $\beta$ is a constant that couples the fields of dark energy and dark matter. The energy density and the pressure of the dark energy are given by

$$
\begin{aligned}
& \rho_{\phi}=\frac{1}{2} \dot{\phi}^{2}+V+6 \xi H \phi \dot{\phi}, \\
& p_{\phi}=\frac{1}{2} \dot{\phi}^{2}-V-2 \xi\left(\phi \ddot{\phi}+\dot{\phi}^{2}+2 H \phi \dot{\phi}\right),
\end{aligned}
$$

respectively, where $V(\phi)$ denotes the dark energy potential density. From (3) - (5) it follows the evolution equation for the energy density of dark energy, namely

$$
\dot{\rho}_{\phi}+3 H\left(\rho_{\phi}+p_{\phi}\right)=-\frac{2 \xi \phi \dot{\phi} \rho}{1-\xi \phi^{2}}+\beta \rho_{d m} \dot{\phi} .
$$

The first term on the right-hand side of (6) is the responsible for the energy transfer from the dark energy to the gravitational field whereas the second refers to an energy transfer to the dark matter field. 
We shall assume that the baryons are non-relativistic particles so that $p_{b}=0$ and that the barotropic equation of state for the radiation field $p_{r}=\rho_{r} / 3$ holds. Hence, the evolution equations for the energy densities of non-interacting baryons and radiation become

$$
\dot{\rho}_{b}+3 H \rho_{b}=0, \quad \dot{\rho}_{r}+4 H \rho_{r}=0,
$$

respectively, and we get from (7) the well-known relationships: $\rho_{b} \propto 1 / a^{3}$ and $\rho_{r} \propto 1 / a^{4}$.

Now by using equations (2) together with (6) and (7) it follows the evolution equation for the energy density of the dark matter field:

$$
\dot{\rho}_{d m}+3 H\left(\rho_{d m}+p_{d m}\right)=-\beta \rho_{d m} \dot{\phi} .
$$

Note that the term on the right-hand side of (8) represents the energy transfer from the dark energy to the dark matter.

\section{APPLICATION}

Two different situations for the dark matter could be analyzed, namely, the one where it is considered as pressureless and the other with a non-vanishing pressure. Here we shall restrict ourselves to the latter case. In order to determine the expression for the dark matter pressure, we recall that in the presence of matter creation the first law of thermodynamics for adiabatic $(d Q=0)$ open systems reads [19]

$$
d Q=d(\rho V)+p d V-\frac{\rho_{d m}+p_{d m}}{n_{d m}} d\left(n_{d m} V\right)=0,
$$

where $V$ denotes the volume and it was supposed that only dark matter creation is allowed. By considering $n_{d m} \propto \rho_{d m}$ and $V \propto a^{3}$ it follows from (9) together with (2) and (6) $-(8)$ :

$$
\frac{2 \xi \phi \dot{\phi} \rho}{1-\xi \phi^{2}}=-\frac{\rho_{d m}+p_{d m}}{n_{d m}}\left(\dot{n}_{d m}+3 H n_{d m}\right)
$$

Now we use again (8) and obtain the following expression for the pressure of the dark matter field

$$
\frac{p_{d m}}{\rho_{d m}}=-\frac{1}{2}-\frac{\beta \dot{\phi}}{6 H} \pm \sqrt{\left(\frac{1}{2}-\frac{\beta \dot{\phi}}{6 H}\right)^{2}+\frac{2 \xi \phi \dot{\phi} \rho}{3 H \rho_{d m}\left(1-\xi \phi^{2}\right)}}
$$

We note that only the plus sign must be chosen in the above equation in order to obtain a positive dark matter pressure. Moreover, we observe that for this case the dark matter pressure vanishes when $\beta=\xi=0$.

We have solved numerically the system of differential equations (3) and (8) as functions of the red-shift $z=1 / a-1$ by using (4), (5), (11) together with $\rho_{b} \propto 1 / a^{3}$ and $\rho_{r} \propto 1 / a^{4}$ and the usual exponential potential $V=V_{0} e^{-\alpha \phi}$, where $\alpha$ and $V_{0}$ are constants. Furthermore, the initial conditions for the energy densities were chosen from the present known values given in the literature [20] for the density parameters $\Omega_{i}(z)=\rho_{i}(z) / \rho(z)$, i.e., $\Omega_{b}(0)=0.04995, \Omega_{r}(0)=5 \times 10^{-5}$ and $\Omega_{d m}(0)=0.23$. The constant $V_{0}$ was determined from

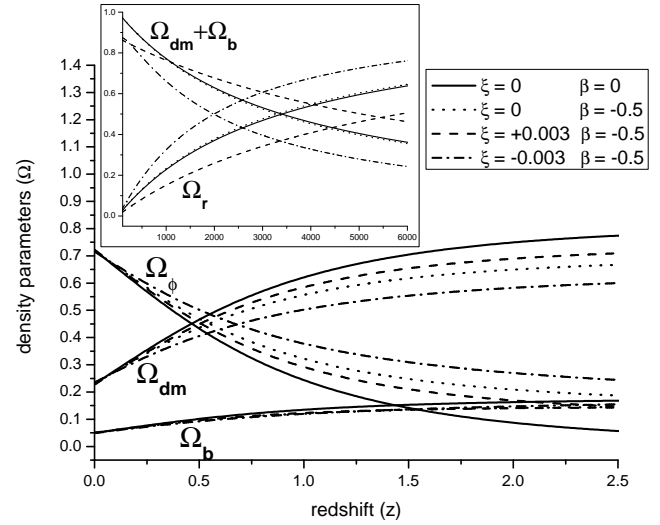

FIG. 1: Evolution of density parameters vs. red-shift.

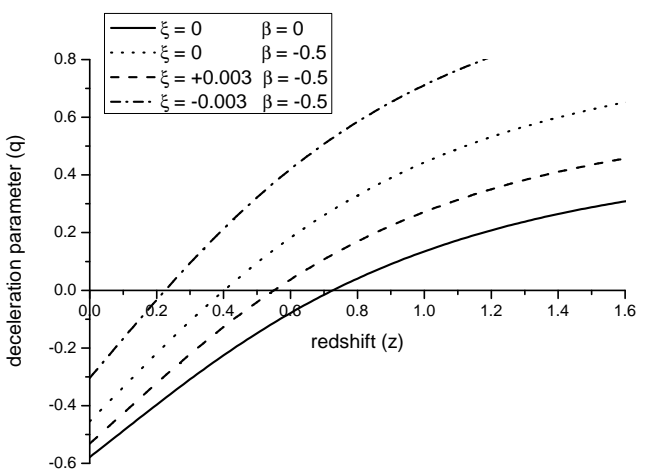

FIG. 2: Deceleration parameter vs. red-shift.

the present value for the dark energy density parameter, i.e., $\Omega_{\phi}(0)=0.72$.

In Fig. 1 we have plotted the red-shift evolution of the density parameters where it was considered a fixed value for the exponent $\alpha$, namely $\alpha=0.1$, and different values for the coupling constants $\beta$ and $\xi$. From this figure we infer that all kinds of coupling delay the growth of dark matter energy density and the decay of dark energy density with respect to the redshift, by a comparison with the uncoupled case, i.e., $\beta=\xi=0$. Also, positive values of $\xi$ imply that the radiation-matter (dark matter plus baryons) equality takes place at higher red-shifts, whereas negative values of $\xi$ at lower ones.

The deceleration parameter $q=-\ddot{a} a / \dot{a}^{2}$ as a function of the red-shift is plotted in Fig. 2. According to current experimental values [21] the decelerated-accelerated transition occurs at $z_{T}=0.46 \pm 0.13$, whereas the present value of the deceleration parameter for the $\Lambda C D M$ case is $q_{0} \approx-0.55$. We note from this figure that the decelerated-accelerated transition shifts from $z_{T} \approx 0.7$ for the uncoupled case $(\beta=\xi=0)$ to $z_{T} \approx 0.2$ for $\beta=-0.5$ and $\xi=0$. Moreover, by keeping a fixed value for $\beta$, positive values for $\xi$ cause later transitions where negative values for $\xi$ imply earlier transitions. This means that, by adjusting the coupling constants in this model, it is possible to have concordance with the experimental data.

In Fig. 3 we have plotted the difference $\mu_{0}$ between the ap- 


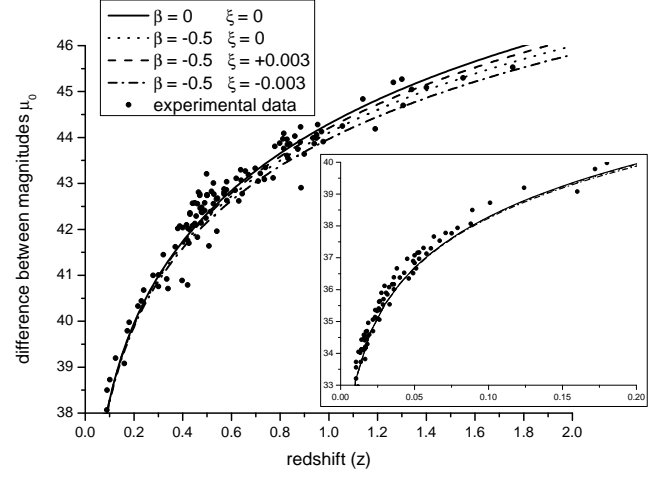

FIG. 3: Difference $\mu_{0}$ vs. red-shift.

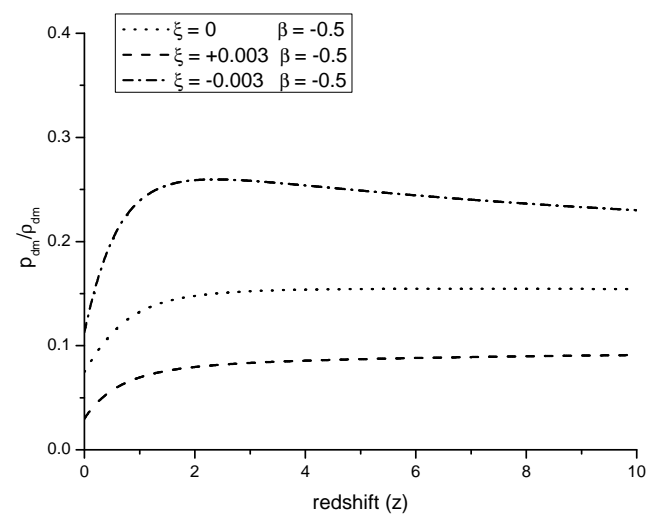

FIG. 4: Dark matter pressure vs. red-shift. parent magnitude $m$ and the absolute magnitude $M$ of a source as a function of the red-shift where the circles represent the experimental values taken from the work by Riess et al. [21] for 185 data points of super-novae of type Ia. The expression for $\mu_{0}$ reads $\mu_{0}=m-M=5 \log d_{L}+25$, where $d_{L}$ is the luminosity distance given in Mpc: $d_{L}=(1+z) c H_{0}^{-1} \int_{0}^{z} d z / H(z)$. We infer from the figure that all cases fit well the experimental data at low red-shifts, but when $\xi$ becomes more negative, the curve goes away from the expected experimental values.

The pressure of the dark matter as function of the red-shift is plotted in Fig. 4. We infer from this figure that for a fixed value of the coupling constant $\beta$, negative values for $\xi$ lead to higher pressure of the dark matter field, and by increasing $\xi$ to positive values the pressure decreases. In all cases the dark matter pressure tend to a constant value for higher red-shifts.

\section{CONCLUSIONS}

The model analyzed here includes two kinds of couplings, namely, the coupling of the scalar field with the curvature scalar and the coupling of the dark matter field with the scalar field. Like the model investigated in the work [12], it has a strong dependence on the initial conditions, since we have solved the system of coupled differential equations by imposing that the slope of the scalar field has small positive values at very high red-shifts, which was a necessary condition to have positive values for the dark energy density. However, as we have shown, there exist some freedom in this model because the two coupling constants can be adjusted in order to fit present and future experimental data.
[1] A. G. Riess et al, Astron. J. 116, 1009 (1998).

[2] S. Perlmutter et al, Astrophys. J. 517, 565 (1999).

[3] S. M. Carroll, Liv. Rev. Rel. 4, 1 (2001).

[4] J. S. Alcaniz, Phys. Rev. D 69, 083521 (2004).

[5] M. S. Madsen, Class. Quant. Grav. 5, 627 (1988).

[6] J. P. Uzan, Phys. Rev. D 59, 123510 (1999).

[7] T. Chiba, Phys. Rev. D 60, 083508 (1999).

[8] L. Amendola, Phys. Rev. D 60, 043501 (1999).

[9] G. Esposito-Farèse and D. Polarski, Phys. Rev. D 63, 063504 (2001).

[10] V. Faraoni, Int. J. Theor. Phys. 40, 2259 (2001).

[11] L. R. Abramo, L. Brenig, E. Gunzig, Phys. Lett. B 549, 13 (2002).

[12] J. B. Binder and G. M. Kremer, Model for a Universe described by a non-minimally coupled scalar field and interacting dark matter (submitted for publication).

[13] J. A. Casas, J. Garcia-Bellido and M. Quiros, Class. Quant. Grav. 9, 1371 (1992).

[14] D. S. M. Alves and G. M. Kremer, JCAP 10, 009 (2004)

[15] M. B. Hoffman, Preprint astro-ph/0307350, (2003).

[16] C. Wetterich, Nucl. Phys. B 302, 645 (1988).

[17] C. Wetterich, Astron. Astrophys. 301, 321 (1995).

[18] L. Amendola, Phys. Rev. D 62, 043511 (2000).

[19] I. Prigogine, J. Géhéniau, E. Gunzig and P. Nardone, Gen. Rel. Grav. 21, 767 (1989).

[20] M. Fukugita and P. J. E. Peebles, Astrophys. J. 616, 643 (2004).

[21] A. G. Riess et al, Astrophys. J. 607, 665 (2004). 